\title{
CONTRIBUTION À LA CONNAISSANCE DE L'INFLUENCE DES GCOULEMENTS TURBULENTS SUR LA DIFFUSION ULTRASONORE PAR UN FOND RUGUEUX
}

\author{
J.P. LONGUEMARD et Y. MEVAL \\ Laboratoire EPAP, Ecole Centrale, Grande Voie des Vignes, F-92290 Châtenay-Malabry, France
}

ABSTRACT

The influence of the turbulence generated by the waves on acoustical diffusion near the seabed is presented. The experiments was made in a hydraulic canal and the surface of the bottom was rough or plan. The peak to peak of the roughness is short or the same than the acoustic wavelength. The acoustical level of the scattering ultrasound is growing when the crest of the waves is at the normal of the transducer. The maxima of the influence of waves on the scattering level is observed when the measures are on a plane bottom submited at the hydrodynamic waves and this for a razance angle of $50^{\circ}$. The geometry of the apparatus and the frequencies used are representative of the acoustical comportment of sound in shallow waters.

RESUME

Ce travail concerne $I^{\prime}$ influence de la houle sur la diffusion acoustique provoquée par un fond 1isse ou rugueux pour des longueurs ondes acoustiques de 1 'ordre de la dimension de la rugosité. Il s'est déroulé dans un bassin avec une houle décrite par la formulation de Stokes par petits fonds. Celle-ci provoque un tourbilion visible par une coloration et mesurable par des moyens optiques. En absence de tourbillon provoqué par la houle, la diffusion des ondes acoustiques sur le fond satisfait aux conditions normales de la diffusion (Rayleigh ; Beckman, Spizzichino). La présence d'une houle induit une augmentation du niveau des ondes diffusées pouvant atteindre 10 dB dans la gamme de fréquences étudiée. Les données acquises en bassin sont représentatives du comportement acoustique en mer (diffusion) à des fréquences beaucoup plus basses (quelques kilohertz sur les ripplemarx et quelques dizaines d'Hertz sur les dunes sous marines).

\section{I - GENERALITES}

Les ondes acoustiques sont émises au centre d'un canal dans lequel circule une houle progressive et se réfléchissent sur le fond. Leurs comportement à 1 'interface dépend de la nature, de la forme, de la rugosité du fond. La houle, au voisinage du fond provoque des tourbillons qui modifient la diffusion acoustique du fond.

Les données acquises dans le canal de 1'Université du Havre concerne soit un fond lisse soit un fond strié soumis à une houle et des fréquences ultrasonores (300 Khz, $1 \mathrm{Mhz}$ ). Les longueurs d'onde varient en regard de la rugosité et sont petites devant les trajets acoustiques. Un système mesurant la houle synchronise 1 'émission de trains d'onde ultrasonore émis verticalement vers le fond par une première sonde piézoélectrique. Ces dernières sont détectées après diffusion sur 1e 
fond à des angles de $10^{\circ}, 30^{\circ}$ et $50^{\circ}$ par une seconde sonde de réception couplée à une chaîne de numérisation et de traitement des données.

\section{II - ANALYSE PHYSIQUE}

Deux comportements physiques sont présents dans cette étude ; les ondes acoustiques et la houle qui modifie les caractéristiques de l'interface eau fond.

\section{II - 1. Comportement acoustique}

Le rapport entre les longueurs d'ondes des ondes ultrasonores émises et les dimensions des sondes fait que le système travaille en champ lointain dans la zone de mesure. L'angle d'ouverture du lobe de rayonnement à $3 \mathrm{~dB}$ pour une sonde varie de $7^{\circ}$ à $15^{\circ}$ pour des fréquences comprises entre 300 et 1 o00 Khz.

Le matériel émet des ondes acoustiques de forme sphérique soit :

$$
P(r, t) \simeq \frac{P_{o}}{r} \exp j(\omega t-k r),
$$

où : $r$ est la longueur du trajet acoustique;

$k$ est le nombre d'onde correspondant à la pulsation $\omega$.

Le signal arrive normalement sur le fond et est renvoyé suivant les lois de Descartes pour un fond plan et suivant les critères de Rayleight pour un fond rugueux $(1)$. L'onde captée par la sonde réception $P_{\text {cap }}$ est définie, pour un élément
de fond, par :

$$
P_{\text {cap }} \simeq \frac{P_{o}}{\left(r_{1} \cdot r_{2}\right)} \exp j\left[\omega t-k\left(r_{1}+r_{2}\right)\right] \cdot \gamma
$$

où : $\gamma(\theta)$ est analogue à un coefficient de réflexion, fonction de 1 'angle de mesure par rapport à la normale;

$r_{1}$ et $r_{2}$ sont les longueurs des trajets incidents et diffusés.

Chaque élément de surface irradié $n$, renvoie une pression vers la sonde réceptrice et la valeur résultante captée vaudra :

$$
P_{c a p} \simeq \sum_{n=1}^{N} \sum_{m=1}^{M} \frac{P_{o}}{\left(r_{1 n} \cdot r_{2 m}\right)} \exp j\left[\omega t-k\left(r_{1 n}+r_{2 m}\right)\right] \sqrt{\gamma_{n m}}(\theta)
$$

La fonction $\gamma_{n m}(\theta)$ satisfait localement aux conditions de renvoi des ondes couramment usitées, soit (2) :

- le rapport entre 1 'écart quadratique moyen de la rugosité $\delta$ et 1a longueur d'onde ;

- La pente de la facette au point de coordonnée $n, m$;

- le coefficient de réflexion sous incidence normale $R_{0}$.

La diffusion, dans cette configuration entre $\theta$ et $\theta+d \theta$ est (2) :

$$
\gamma(\theta)=R_{0} 2 \pi^{4}\left(\frac{a}{r}\right)^{2}\left(\frac{\delta}{r}\right)^{2}(\cos \theta+1)^{4} \sin \theta \exp -\left(\frac{\pi_{a} \sin \theta}{r}\right)^{2}
$$

où : $a=\sqrt{\delta} / m$ (m est la pente et $\delta$ la rugosité) ;

$h_{o}$ est le coefficient de réflexion sans rugosité. 


\section{II - 2. Comportement_de 1a_houloule_dans_le_canal 1}

$D^{\prime}$ une amplitude de $24 \mathrm{~mm}$ et une longueur d'onde $L=1,46 \mathrm{~m}$, elle est supposée représenter des effets analogues à ceux de la houle par petits fonds. Les caractéristiques de cette dernière sont fixes et l'on a préféré faire varier la fréquence acoustique pour apprécier 1 'influence relative des conditions hydrodynamiques. E11e est représentée par la formulation de Stokes.

Les mouvements de particules au voisinage du fond dont 1'amplitude dépend de la profondeur ( $H$ ) du canal, de I'amplitude de la houle (A) et de longueur d'onde $L$ sont tels que :

- I'élongation horizontale vaut :

$$
S_{h}=A \frac{C h 2 \pi \frac{y+H}{L}}{\operatorname{sh} 2 \pi \frac{H}{L}}, S_{h} \text { tend vers } \frac{A L}{2 \pi H} \text { quand } \frac{L}{H} \text { croit }
$$

- 1 'élongation verticale vaut :

$$
S_{V}=A \frac{\operatorname{Ch} 2 \pi \frac{y+H}{L}}{\operatorname{sh} 2 \pi \frac{H}{L}}, S_{V} \text { tend vers } A\left(1+\frac{y}{H}\right) \text { quand } \frac{L}{H} \text { croit. }
$$

Des turbulences sont induites sur 1es rides, avec une amplitude de 1 'ordre du pas de ces dernières. Des zones de turbulence intense apparaissent à la crête des rides. Ces dernières sont à même de provoquer une modification notable (découlement) du fluide donc une possibilité de diffusion des ondes acoustiques à la crête des tourbillons et non plus sur 1'interface lui-même.

\section{III - ANALYSE DES RESULTATS}

L'analyse rigoureuse du comportement physique est complexe aussi nous avons préféré travailler par comparaison entre un fond rugueux et un même fond mais lisse soumis aux mêmes conditions hydrodynamiques. Le comportement le plus significatif fut observé à $300 \mathrm{Khz}$.

Une influence importante de la houle prend naissance lorsque la crête de la houle passe à $1^{\prime}$ aplomb de la sonde émettrice pour des angles d'incidence de $10^{\circ}$ et $30^{\circ}$. Le même phénomène a lieu pour $\theta=50^{\circ}$ mais avec un déphasage de $30 \%$ de 1 a longueur d'onde. L'effet relatif le plus sensible a lieu pour un fond lisse quelque soit la phase de la houle.

Cette constatation s'explique par le fait que sans rugosité, le fond ne diffuse pas les ondes acoustiques. Les turbulences engendrées par la houle ont, dans ce cas, une action significative par la réaction d'une rugosité artificielle.

Ce tourbillon produit alors une augmentation du niveau de plus de $10 \mathrm{~dB}$. En éclairant plusieurs rides, les transducteurs provoquent une diffusion accrue dans toutes les directions et homogénéisent les niveaux reçus surtout à $30^{\circ}$ par rapport à la houle seule.

La phase de la houle par rapport aux sondes acoustiques en agissant sur la position dans 1 'espace du tourbillon, provoque indirectement un accroissement du niveau acoustique.

\section{IV - CONCLUSION}

L'influence de la houle est d'autant plus importante que le fond est lisse, ce qui est $d u$ au fait que les ridules produisent elles-mêmes une partie des ondes diffuses. L'accroissement du niveau diffusé, pour $\theta$ valant $50^{\circ}$ dépasse largement $10 \mathrm{~dB}$ à 300 et à $1000 \mathrm{Khz}$. Ce phénomène moins sensible pour $\theta=10$ degrés (gain de 
1 'ordre de 3 à $6 \mathrm{~dB}$ ) disparaît en fait vers 30 degrés lorsqu'il y a des ridules.

Ces quelques résultats quoique fragmentaires confirment qu'il serait possible d'étudier les turbulences hydrauliques au niveau d'un interface par analyse de la réponse acoustique en particulier par un système multisonde en vue de définir les points de naissance des turbulences. L'appréciation de la dimension de celle-ci est également possible en utilisant plusieurs fréquences acoustiques.

Ces résultats sont représentatifs d'un effet dû à la houle par petits fonds à des fréquences acoustiques beaucoup plus basses.

$\mathrm{V}$ - BIBLIOGRAPHIE

(1) - P. BECHMAN, A. SPIZZICHINO : The scattering of electromagnetic waves from rough surface. Pergamon Press 1963.

(2) - H.E. BENNET, J.O. PORTENS : Relation between surface roughness and specalar reflectance at normal incidence. Jour. of the Optical Society of America. Vol 54. Nu. 2.2. 1961 .

(3) - P.A. CROWTHER : Some statistic of the sea bed and Acoustic scattering therefrom. Proceedings of an Institute of acoustic "Acoustics and the sea bed"1983.

(4) - P.H. THORNE, N.G. PACE : Broad Band studies of Acoustics scattering from a rough mode1 surface. Proceedings of an Institute of Acoustics "Acoustics and the sea bed". 1983.

TABLEAU : Niveau acoustique mesuré, proportionnel à Peap à $300 \mathrm{Khz}$ et $1 \mathrm{Mhz}$

\begin{tabular}{|c|c|c|c|c|c|}
\hline & $\begin{array}{l}\text { angle } \\
\text { de } \\
\text { mesure }\end{array}$ & sans houle & $\begin{array}{l}\text { avec houle } \\
\text { sans retard }\end{array}$ & $\begin{array}{l}\text { avec houle } \\
\text { avec retard } \\
\text { de } 130 \%\end{array}$ & $\begin{array}{l}\text { avec houle } \\
\text { avec retard } \\
\text { de } 160 \%\end{array}$ \\
\hline \multirow{3}{*}{$\begin{array}{l}\text { PLAQUE ALUMINTUM } \\
\text { LISSE POUR UNE } \\
\text { FREQUENCE } \\
\text { DE } 300 \mathrm{KHZ} \mathrm{A} \\
\end{array}$} & $10^{\circ}$ & $746 \mathrm{mV}$ & $2,30 \mathrm{~V}$ & $2,14 \mathrm{~V}$ & $1,48 \mathrm{~V}$ \\
\hline & $30^{\circ}$ & $780 \mathrm{mV}$ & $936 \mathrm{mV}$ & $1,74 \mathrm{~V}$ & $1,56 \mathrm{~V}$ \\
\hline & $50^{\circ}$ & $136 \mathrm{mV}$ & $616 \mathrm{mV}$ & $576 \mathrm{mV}$ & $560 \mathrm{mV}$ \\
\hline \multirow{3}{*}{$\begin{array}{l}\text { PLAQUE ALUMINIUM } \\
\text { AVEC DES RIDULES } \\
\text { POUR UNE FREQUENCE } \\
\text { DE } 300 \mathrm{KHZ} \mathrm{B}\end{array}$} & $10^{\circ}$ & $832 \mathrm{mV}$ & $1,68 \mathrm{~V}$ & $1,84 \mathrm{~V}$ & $1 \mathrm{~V}$ \\
\hline & $30^{\circ}$ & $1,36 \mathrm{~V}$ & $1,39 \mathrm{~V}$ & $1,37 \mathrm{~V}$ & $1,24 \mathrm{~V}$ \\
\hline & $50^{\circ}$ & $1,41 \mathrm{~V}$ & $1,12 \mathrm{~V}$ & $1,41 . V$ & $1,41 \mathrm{~V}$ \\
\hline \multirow{3}{*}{$\begin{array}{c}\text { PLAQUE ALUMINIUM } \\
\text { AVEC DES RIDULES } \\
\text { POUR UNE FREQUENCE } \\
\text { DE } 1 \text { MHZ C }\end{array}$} & $10^{\circ}$ & $10.6 \mathrm{~V}$ & $10 \mathrm{~V}$ & $9,3 \mathrm{~V}$ & $8,9 \mathrm{~V}$ \\
\hline & $30^{\circ}$ & $2,3 \mathrm{~V}$ & $2,02 \mathrm{~V}$ & $2,04 \mathrm{~V}$ & $1,67 \mathrm{~V}$ \\
\hline & $50^{\circ}$ & $896 \mathrm{mV}$ & $944 \mathrm{mV}$ & $932 \mathrm{mV}$ & $854 \mathrm{mV}$ \\
\hline $\begin{array}{l}\text { PLAQUE ALUMINIUM } \\
\text { LISSE POUR UNE } \\
\text { FREQUENCE } \\
\text { DE } 1000 \mathrm{KHZ} \mathrm{D}\end{array}$ & $50^{\circ}$ & $40 \mathrm{mV}$ & $146 \mathrm{mV}$ & $204 \mathrm{mV}$ & $99 \mathrm{mV}$ \\
\hline
\end{tabular}

